\title{
Imaging Venous Angiomas
}

Venous angiomas or venous malformations are probably not true malformations. They appear to be variants or developmental anomalies that provide anomalous venous drainage of normal brain parenchyma. (Glendhill et al., 2000). Their incidence is $\sim 3 \%$ at autopsy. They are frequent incidental findings on post-contrast studies of the brain.

\section{VENOUS ANGIOMAS}

The following sequences are useful for evaluating venous angiomas:

1. Three-plane positioning scout.

2. Transverse $T_{1}$-weighted post-gadolinium with flow compensation.

3. Coronal $T_{1}$-weighted post-gadolinium coronal with flow compensation.

4. Sagittal post- $T_{1}$-weighted gadolinium with flow compensation.

5. MRV (magnetic resonance venography).

Although the actual scan time is $15 \mathrm{~min}$ and $46 \mathrm{sec}$, because of setup and injection time, we tell the patient that the examination will take approximately one-half hour. Except for the three-plane positioning scout, if the patient has recently had a routine cranial MR examination, we perform only these sequences necessary to complete this protocol. (As an example, a routine cranial MR consists of sequences 1 to 5 in UNIT A5.1, without using contrast agents.) It is actually more common for us to find venous angiomas incidentally on contrasted MRI examinations than to have found them on CT (computed tomography), angiography, or previous MRI, and to look for them prospectively. In the less common situation where the patient has not had a routine cranial MR, we add $T_{2}$-weighted sequences (without contrast agents) to provide a more complete examination. For example, one can run a $T_{2}$-weighted sequence whose imaging parameters are listed in Table A2.2.5, with imaging plane set to transverse and with a possible change of number of slices or slice thickness in order to cover the entire brain. Figures A2.3.1 and A2.3.2 are example images.

Table A2.3.1 lists the hardware necessary to perform the procedure, along with appropriate parameters. The available gradient strength will depend on the scanner, and the echo times given in other tables below may be varied accordingly (the smaller the gradient strength, the longer the echo time for a particular scan).

NOTE: Be sure that technologists and nurses have immediate access to any emergency equipment that may be relevant to a given study, or that may be needed for a particular patient, such as a crash cart or oxygen.

\section{Materials}

Normal saline $(0.9 \% \mathrm{NaCl})$

Sterile extravascular contrast agent (e.g., Magnevist, Ominiscan, Prohance)

\section{Set up patient and equipment}

1. Interview (screen) the patient to assess for contraindications such as cardiac pacemaker, implanted mechanical devices, and/or ferromagnetic materials. Also, determine if the patient will need sedation, necessitating the need for appropriate monitoring equipment.

Each patient or legal guardian must sign a screening form (see, e.g., APPENDIX 1) before the patient is brought into the exam area.
BASIC

PROTOCOL

Cerebral Venous

Lesions

A2.3.1

Supplement 9 


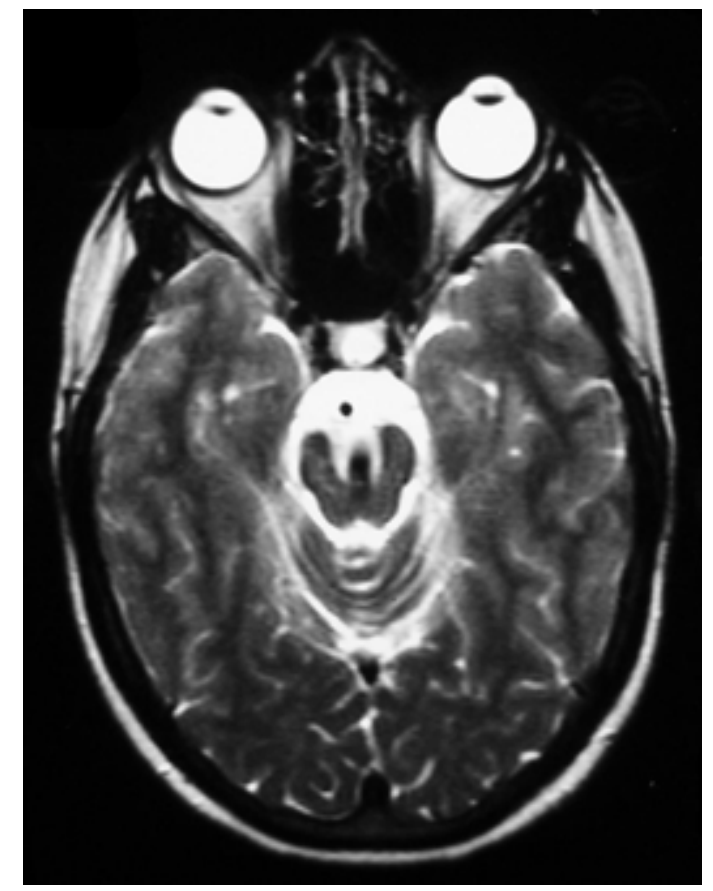

Figure A2.3.1 A transverse $T_{2}$-weighted image demonstrates abnormal "flow void" through the level of the pons just posterior to the smaller but normal flow void in the basilar artery in the prepontine cistern.

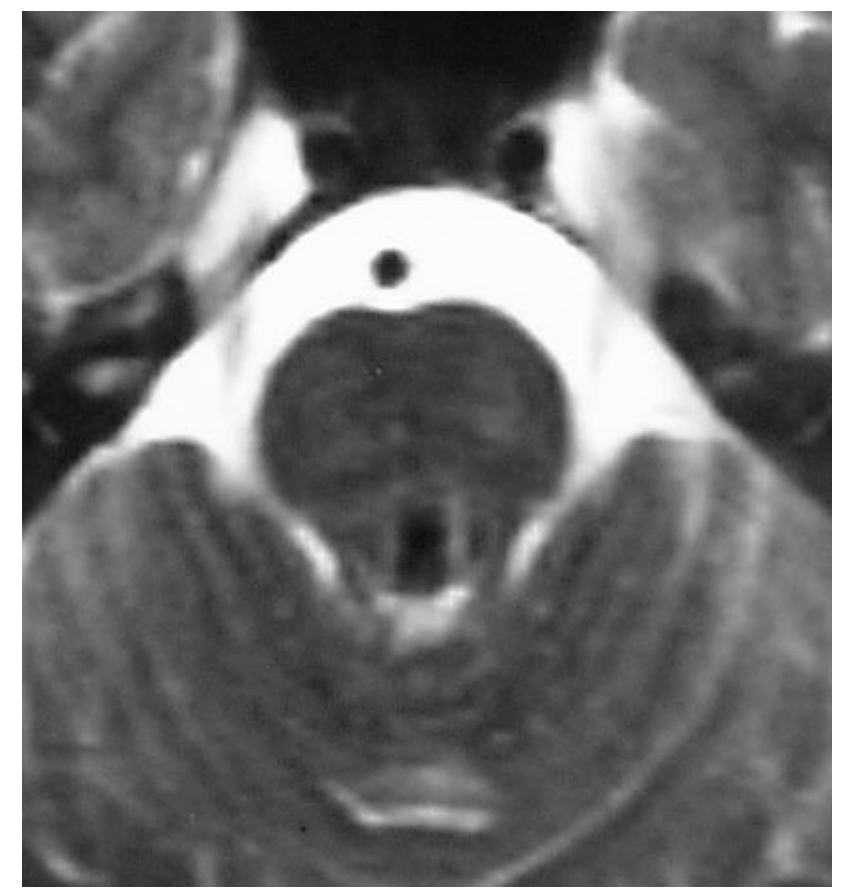

Figure A2.3.2 Close-up of the transverse $T_{2}$-weighted image through the pons at lower level (caudal) to the image shown in Figure A2.3.1. This shows continuation of the same abnormal "flow void" seen on the image of the same patient as shown Figure A2.3.1. 
Table A2.3.1 Equipment Specifications Needed to Perform the Following Imaging Sequences

\begin{tabular}{ll}
\hline $\begin{array}{l}\text { Coil type } \\
\text { Field strength } \\
\text { Gradient strength }\end{array}$ & $\begin{array}{l}\text { Circularly polarized head coil } \\
1.5 \mathrm{~T} \\
\text { Minimum of } 15 \mathrm{mT} / \mathrm{m} \text { (or whatever the } \\
\text { system permits) }\end{array}$ \\
$\begin{array}{l}\text { Positioning cushion or } \\
\text { head stabilizers }\end{array}$ & $\begin{array}{l}\text { Yes } \\
\text { Knee cushion }\end{array}$ \\
$\begin{array}{l}\text { Pulse oximeter } \\
\text { Use of contrast agents }\end{array}$ & $\begin{array}{l}\text { Yes patient requires sedation } \\
\text { Yes }\end{array}$ \\
\hline
\end{tabular}

The presence of ferromagnetic materials may be a health hazard to the patient while in the magnetic field and/or adversely affect image quality. To determine the safety of scanning such ferromagnetic materials see Shellock (2001).

The presence offerromagnetic materials in the globe of the eye is contraindicated for MRI. Patients with prior metal exposure to the eye should have plain $X$ rays of the orbital area to ensure that all metal has been removed, prior to placing them in the magnetic field.

2. If the procedure is a research protocol, have the patient sign any necessary consent form.

3. Request that the patient change into a gown and remove all personal items such as, jewelry, hearing aids, glasses, any metal, etc., prior to entering the MRI scan room. All personal belongings should be secured during the examination.

4. Have the patient wash off any mascara and other makeup to avoid local tissue heating and image artifacts.

5. Explain the procedure to the patient and record relevant clinical history. Ensure that the patient understands what is expected and ask him or her if he or she has any questions; answer appropriately.

6. Set up the exam room by providing a clean exam (scanning) table and securing the circularly polarized $(\mathrm{CP})$ head coil to the table.

7. Escort the patient to the MR examination room and ask him or her to lie down on the table accordingly with respect to the exam to be performed. Review the following items with the patient:

a. Provide earplugs or headphones to the patient to minimize the loud knocking noise that will be produced by the gradients but ensure him or her that he or she will still be able to hear you.

b. Provide the patient with a safety squeeze-bulb and demonstrate how it works; explain to the patient when to use the squeeze-bulb (i.e., if they need assistance during the exam).

c. Explain to the patient that you will be talking to him or her between imaging sequences when the loud knocking noise stops.

d. Explain to the patient that it is imperative that he or she remain motionless during the loud knocking noise to ensure good results; also explain that he or she should not reposition his or her body (especially head) between imaging sequences.

e. Provide the patient with an approximate time that the examination will take. If this entire protocol will be followed, we suggest that the exam will take approximately half an hour.

f. Nevertheless, the patient may call out at any time if he or she feels it necessary.

Cerebral Venous

Lesions

A2.3.3

Supplement 9 
8. Either before or right after the patient lies down, set up any triggering devices or other monitoring equipment to be used.

9. Establish an intravenous line from which the contrast agent can be injected, and attach this line securely to the patient so that movement into or out of the magnet will not pull at the patient's arm.

It is preferable to insert the line prior to imaging and to leave the patient in the magnet, so that there is no intervening motion between the scans run before contrast agent injection and those run after injection.

10. Center the patient in the head coil, at the region where the key information is desired. Make sure that the head is constrained to prevent motion, especially if high-resolution scans are to be run. Secure the patient's head with positioning sponges or head stabilizers.

Generally, the patient's head is fixed so that it is horizontal (not tilted) and lies along the axis of the patient table; other positions may be appropriate depending on the needs at hand.

11. If needed, place a pillow or other support under the knees to make the patient more comfortable.

12. Use the centering light to position the patient's nasion and advance the patient into the center of the magnet.

Once this step has been performed, so long as the patient does not move on the table, the table itself can be moved and then returned to the same position as before without jeopardizing the positioning of one scan relative to another.

13. If the patient is unable to hold still, provide appropriate sedation.

\section{Sequence 1: Three-plane positioning scout}

14. To validate the patient's position and to have a reference to prescribe successive imaging sequences, acquire a three-plane orthogonal scout sequence. See Table A2.3.2 for specific parameters.

Most MR scanners can be programmed to acquire the scout automatically after coil tuning or after the patient has been placed in isocenter (for systems that do not require tuning). This runs in $7 \mathrm{sec}$ and is used to position the remainder of the sequences. It is particularly useful to correct off-axis positioning in the coronal plane.

\section{Sequence 2: Transverse post-gadolinium with flow compensation}

15. Display both the sagittal and transverse scout images in two separate quadrants on the scan monitor. Change imaging parameters to those listed in Table A2.3.3. Position slices to midline of the sagittal scout image. Adjust position of field of view off transverse scout image.

16. Leaving the patient in the magnet, inject the contrast agent either by hand or using a mechanical injector. Observe the injection to insure there is no extravasation of the contrast agent. Flush the line with $10 \mathrm{ml}$ of sterile normal saline. Begin the scan as soon as the injection is completed.

Alternatively, the scanning table may be advanced out of the magnet for the injection, but the patient must remain in place.

A dose of $0.1 \mathrm{mmol} / \mathrm{kg}$ of contrast agent is usually given.

17. Instruct the patient to remain motionless, as the scan will begin and last for $\sim 5$ min.

Imaging Venous Angiomas

A2.3.4 
Table A2.3.2 Imaging Parameters for Sequence 1 (Scout Sequence)

\begin{tabular}{ll}
\hline Patient position & Supine \\
Scan type & Gradient echo \\
Imaging plane (orientation) & Transverse, sagittal, and coronal \\
Central slice or volume center & Nasion \\
Echo time $\left(T_{\mathrm{E}}\right)$ & $6 \mathrm{msec}$ \\
Repeat time $\left(T_{\mathrm{R}}\right)$ & $15 \mathrm{msec}$ \\
Flip angle $(\mathrm{FA})$ & $30^{\circ}$ \\
Fields of view $\left(\mathrm{FOV}, \mathrm{FOV}_{\mathrm{y}}\right)$ & $300 \mathrm{~mm}, 300 \mathrm{~mm}$ \\
Resolution $(\Delta x, \Delta y)$ & $1.17 \mathrm{~mm}, 2.34 \mathrm{~mm}$ \\
Number of data points collected $\left(N_{\mathrm{x}}, N_{\mathrm{y}}\right)$ & 256,128 \\
Slice thickness $(\Delta z)$ & $8 \mathrm{~mm}$ \\
Number of slices & $3($ one per orientation $)$ \\
Slice gap & Not applicable \\
Number of acquisition $\left(N_{\mathrm{acq}}\right)$ & 1 \\
Swap read and phase encoding & No \\
Slice location & Not applicable \\
Saturation pulses & Not applicable \\
Scan time & 7 sec \\
\hline
\end{tabular}

Table A2.3.3 Imaging Parameters for Sequence 2 (Transverse $T_{1}$-Weighted Spin Echo with Flow Compensation)

\begin{tabular}{ll}
\hline Patient position & Supine \\
Scan type & Spin echo \\
Imaging plane (orientation) & Transverse \\
Central slice or volume center & Slices centered to midline on sagittal \\
& scout \\
Echo time $\left(T_{\mathrm{E}}\right)$ & $17 \mathrm{msec}$ \\
Repeat time $\left(T_{\mathrm{R}}\right)$ & $532 \mathrm{msec}$ \\
Flip angle $(\mathrm{FA})$ & $90^{\circ}$ \\
Fields of view $(\mathrm{FOV}$ & $\left.\mathrm{FOV}_{\mathrm{y}}\right)$ \\
& $230 \mathrm{~mm}, 230 \mathrm{r}$ mm, with $r=3 / 4$ \\
Resolution $(\Delta x, \Delta y)$ & $($ rectangular field of view) \\
Number of data points collected $\left(N_{\mathrm{x}}, N_{\mathrm{y}}\right)$ & $0.90 \mathrm{~mm}, 0.90 \mathrm{~mm}$ \\
& $256,256 r$, with $r=3 / 4$ (rectangular \\
Slice thickness $(\Delta z)$ & field of view) \\
Number of slices & $5 \mathrm{~mm}$ \\
Slice gap & 19 \\
Number of acquisitions $\left(N_{\mathrm{acq}}\right)$ & $1.5 \mathrm{~mm}(-30 \%)$ \\
Read direction & 2 \\
Slice locations & Anterior-posterior \\
Flow compensation & Vertex to skull base \\
Saturation pulses & Yes \\
Slice series & None \\
Scan time & Interleaved \\
\hline & $3 \mathrm{~min}, 26$ sec \\
\hline
\end{tabular}

Cerebral Venous

Lesions

A2.3.5 


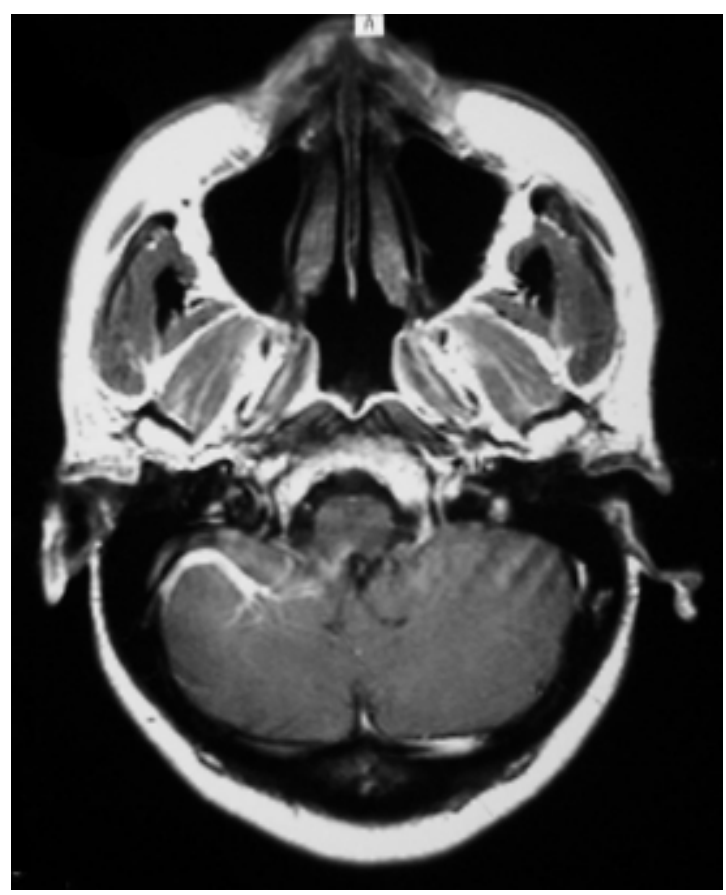

Figure A2.3.3 A post-contrast transverse $T_{1}$-weighted image demonstrates typical venous angioma of the right cerebellar hemisphere.

Table A2.3.4 Imaging Parameters for Sequence 3 (Coronal $T_{1}$-Weighted Spin Echo with Flow Compensation)

\begin{tabular}{ll}
\hline Patient position & Supine \\
Scan type & Spin echo \\
Imaging plane (orientation) & Coronal \\
Central slice or volume center & Slices centered to midline on sagittal \\
& scout \\
Echo time $\left(T_{\mathrm{E}}\right)$ & $17 \mathrm{msec}$ \\
Repeat time $\left(T_{\mathrm{R}}\right)$ & $532 \mathrm{msec}$ \\
Flip angle $(\mathrm{FA})$ & $90^{\circ}$ \\
Fields of view $\left(\mathrm{FOV}_{\mathrm{x}}, \mathrm{FOV}_{\mathrm{y}}\right)$ & $230 \mathrm{~mm}, 230 \mathrm{rmm}$, with $r=3 / 4$ \\
& $($ rectangular field of view) \\
Resolution $(\Delta x, \Delta y)$ & $0.90 \mathrm{~mm}, 0.90 \mathrm{~mm}$ \\
Number of data points collected $\left(N_{\mathrm{x}}, N_{\mathrm{y}}\right)$ & $256,256 r$, with $r=3 / 4$ (rectangular \\
& field of view) \\
Slice thickness $(\Delta z)$ & $5 \mathrm{~mm}$ \\
Number of slices & 19 \\
Slice gap & $1.5 \mathrm{~mm}(-30 \%)$ \\
Number of acquisitions $\left(N_{\mathrm{acq}}\right)$ & 2 \\
Read direction & Right-left \\
Slice locations & Equidistant between anterior-posterior \\
& brain \\
Flow compensation & Yes \\
Saturation pulses & None \\
Slice series & Interleaved \\
Scan time & 3 min, 26 sec \\
\hline
\end{tabular}


Table A2.3.5 Imaging Parameters for Sequence 4 (Sagittal $T_{1}$-Weighted Spin Echo with Flow Compensation)

Patient position

Scan type

Imaging plane (orientation)

Central slice or volume center

Echo time $\left(T_{\mathrm{E}}\right)$

Repeat time $\left(T_{\mathrm{R}}\right)$

Flip angle (FA)

Fields of view $\left(\mathrm{FOV}_{\mathrm{x}}, \mathrm{FOV}_{\mathrm{y}}\right)$

Resolution $(\Delta x, \Delta y)$

Number of data points collected $\left(N_{\mathrm{x}}, N_{\mathrm{y}}\right)$

Slice thickness $(\Delta z)$

Number of slices

Slice gap

Number of acquisitions $\left(N_{\text {acq }}\right)$

Read direction

Slice locations

Flow compensation

Saturation pulses

Slice series

Scan time
Supine

Spin echo

Sagittal

Slices centered to midline on transverse scout

$17 \mathrm{msec}$

$633 \mathrm{msec}$

$90^{\circ}$

$210 \mathrm{~mm}, 210 r \mathrm{~mm}$, with $r=3 / 4$ (rectangular field of view)

$0.82 \mathrm{~mm}, 0.82 \mathrm{~mm}$

256, 256r, with $r=3 / 4$ (rectangular field of view)

$5 \mathrm{~mm}$

25

$1.5 \mathrm{~mm}(-30 \%)$

1

Anterior-posterior

Equidistant between left and right brain Yes

None

Interleaved

$2 \mathrm{~min}, 4 \mathrm{sec}$

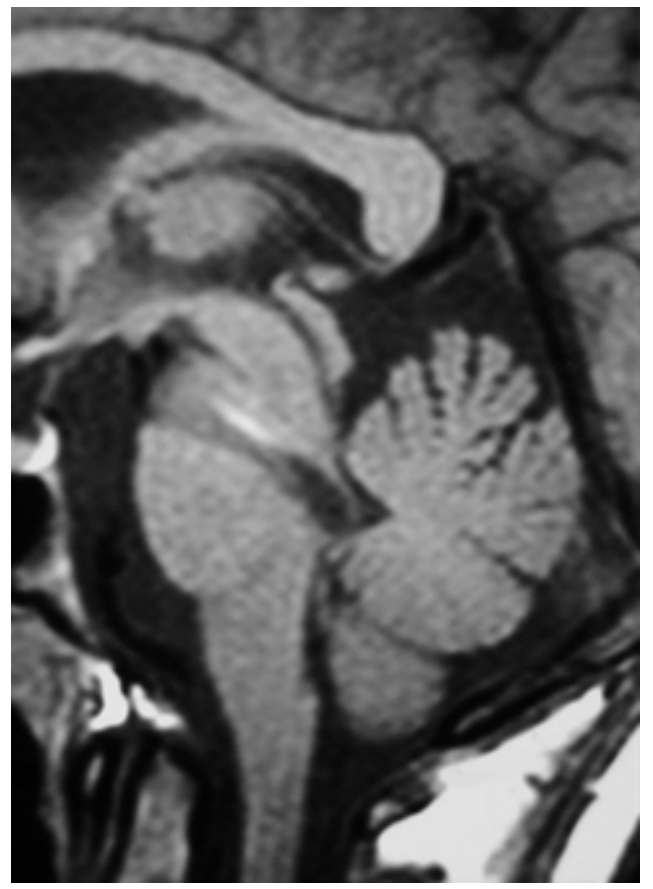

Figure A2.3.4 Close-up of a sagittal $T_{1}$-weighted image shows large abnormal area of low signal extending through the pons posteriorly and inferiorly toward the fourth ventricle. This corresponds to the abnormality (vascular flow void) seen on images in Figure A2.3.1. and Figure A2.3.2.

Cerebral Venous Lesions 


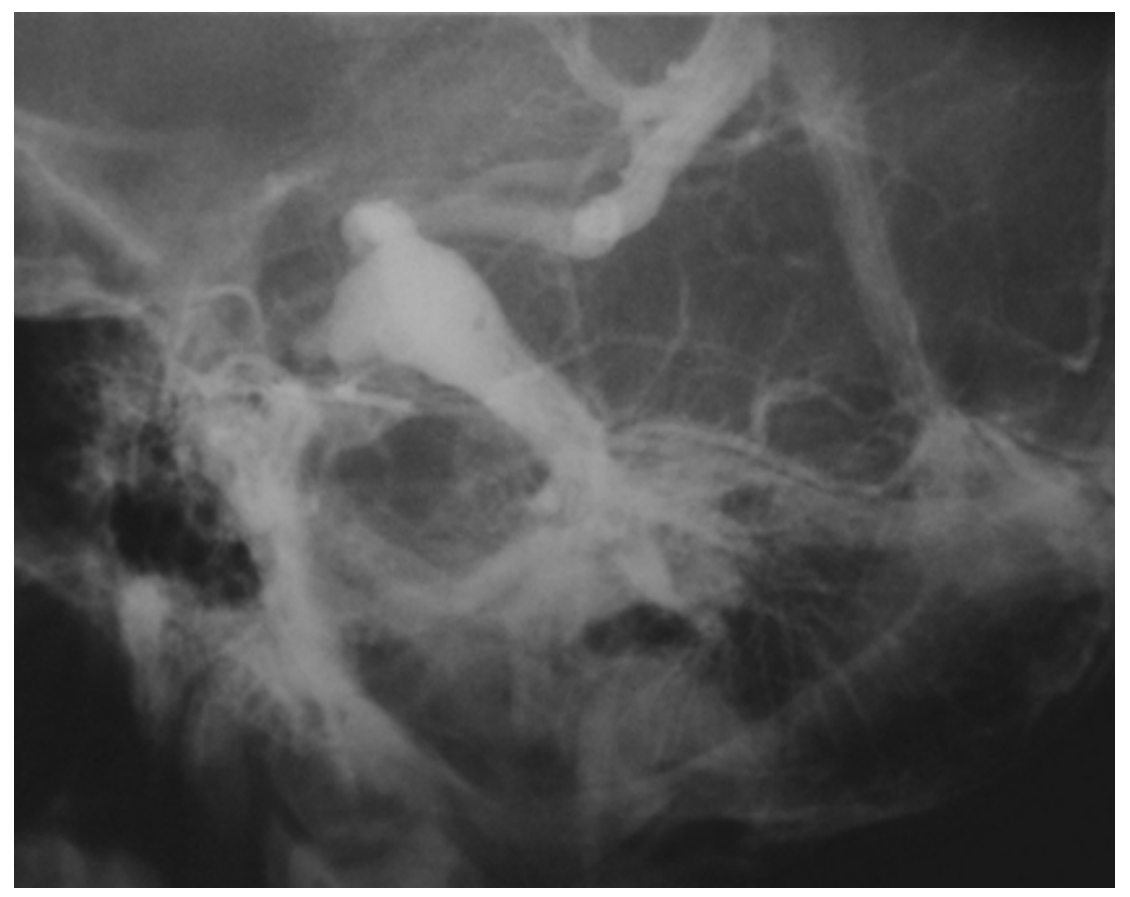

Figure A2.3.5 Close-up lateral view of the venous phase from conventional angiogram demonstrates a large venous angioma in a transpontine location. This contrast-enhanced vascular structure corresponds to the abnormality seen on the previous three images (Figures A2.3.1, A2.3.2, and A2.3.4).

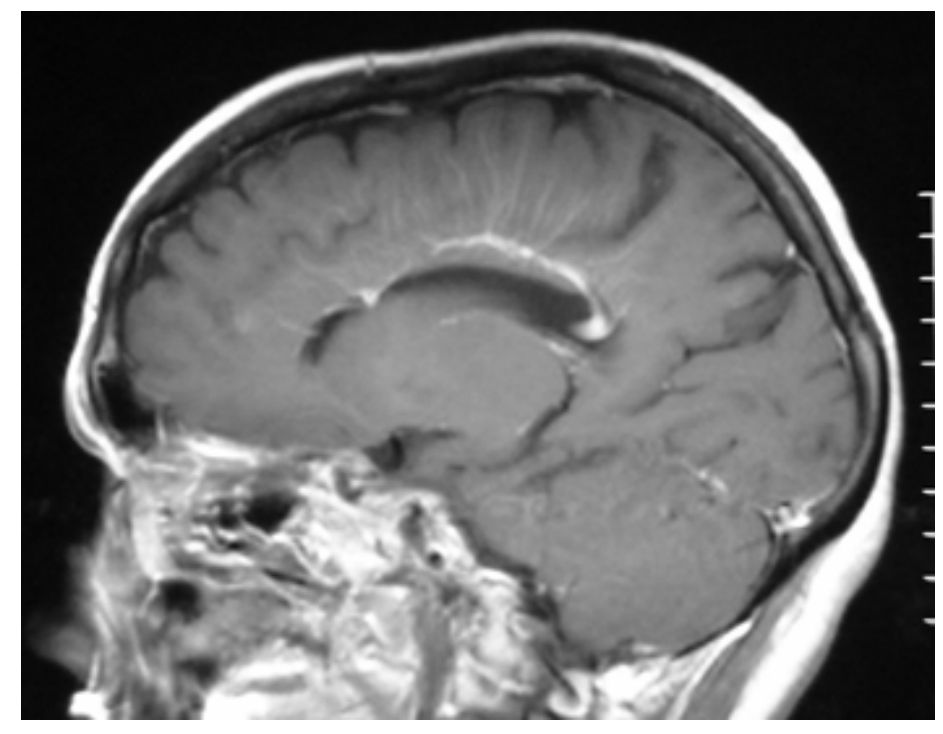

Figure A2.3.6 A sagittal $T_{1}$-weighted image with gadolinium shows venous angioma extending in periventricular area from the anterior portion of the lateral ventricle posteriorly into the lateral ventricle. From same patient as image shown in Figure A2.3.7; look back at the previous image to see some of the flow voids now filled with a contrast agent. 


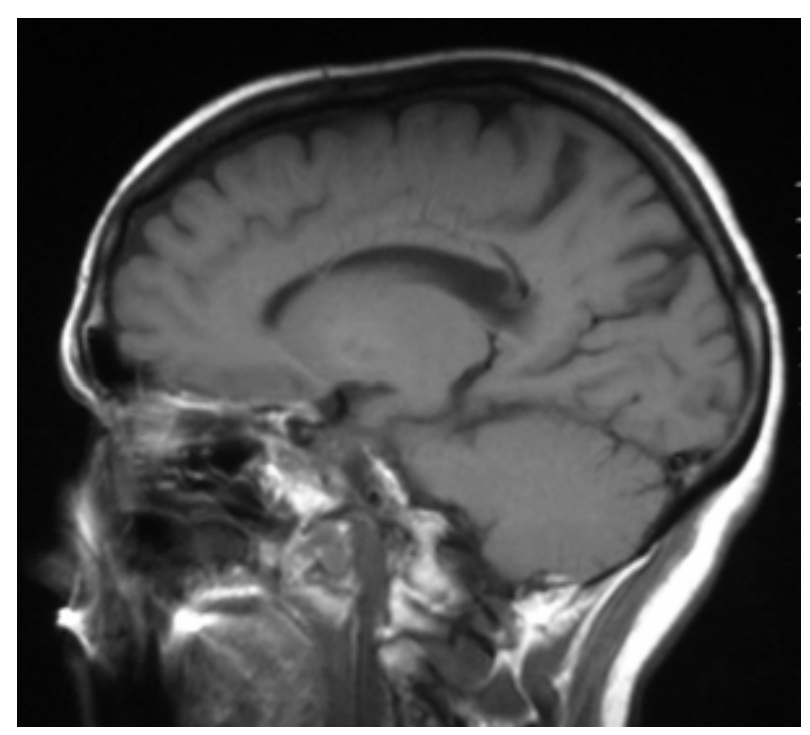

Figure A2.3.7 A sagittal $T_{1}$-weighted image shows abnormal flow voids paralleling superior aspect of the ventricle. It may be easy to overlook venous angiomas on $T_{1}$-weighted images without gadolinium.

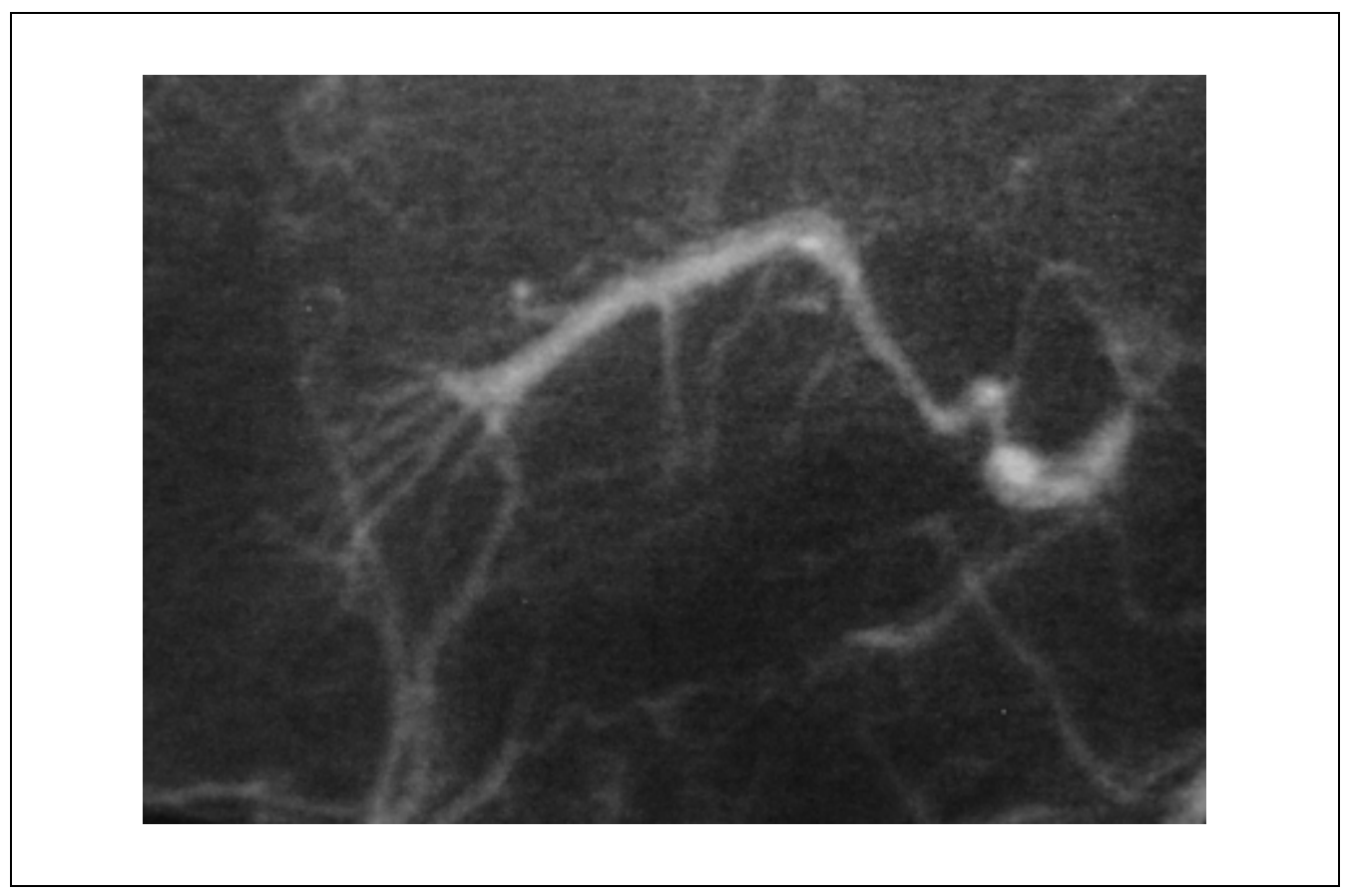

Figure A2.3.8 Coned lateral view of the venous phase from conventional cerebral angiogram. This demonstrates a typical venous angioma with a "carrot's top" appearance and mirrors the appearance seen on a sagittal MRI or MRV. Multiple radicles converge on an anomalous dilated vein which in turn drains into dilated central venous structures.

Cerebral Venous

Lesions 
Table A2.3.6 Imaging Parameters for Sequence 5 Time-of-Flight MRV of the Sagittal Sinus

\begin{tabular}{ll}
\hline Patient position & Supine \\
Scan type & 2-D gradient echo \\
Imaging plane & Sagittal \\
Central slice or volume center & Slices centered to midline on \\
& transverse scout \\
Echo time $\left(T_{\mathrm{E}}\right)$ & $9 \mathrm{msec}$ \\
Repeat time $\left(T_{\mathrm{R}}\right)$ & $30 \mathrm{msec}$ \\
Flip angle $(\mathrm{FA})$ & $50^{\circ}$ \\
Field of view $\left(\mathrm{FOV}_{\mathrm{x}}, \mathrm{FOV}_{\mathrm{y}}\right)$ & $210 \mathrm{~mm}, 210 \mathrm{~mm}$ \\
Resolution $(\Delta x, \Delta y)$ & $0.82 \mathrm{~mm}, 0.82 \mathrm{~mm}$ \\
Number of data collected $\left(N_{\mathrm{x}}, N_{\mathrm{y}}\right)$ & 256,256 \\
Slice thickness $(\Delta z)$ & $3 \mathrm{~mm}$ \\
Number of slices & 64 \\
Slice gap & $0.34 \mathrm{~mm}$ \\
Number of acquisitions $\left(N_{\mathrm{acq}}\right)$ & 1 \\
Swap read and phase encoding & No \\
Slice locations & Angle sagittal to coronal, $-12^{\circ}$ (to \\
& reduce in-plane saturation) off \\
Saturation pulses & transverse scout image \\
& Yes (one angled through facial sinus \\
Scan time & area off sagittal scout image, 60-mm \\
thick)
\end{tabular}

${ }^{a}$ If the number of slices is set to be 1 , the scan time is $7.8 \mathrm{sec}$.

18. Run sequence 2 according to Table A2.3.3.

An example image is shown in Figure A2.3.3.

This is the basic post-contrast, flow-compensated sequence which will be performed in transverse, coronal, and sagittal planes. If a venous angioma is present, these three sequences will probably define it with the least artifact.

Sequence 3: Coronal post-contrast $T_{1}$-weighted with flow compensation

19. Run sequence 3 according to Table A2.3.4.

\section{Sequence 4: Sagittal post-contrast $T_{1}$-weighted with flow compensation}

20. Position slices to midline of the transverse scout image. Run sequence 4 according to Table A2.3.5.

Some example images are shown in Figure A2.3.4, Figure A2.3.5, Figure A2.3.6, Figure A2.3.7, and Figure A2.3.8.

The term used by Siemens for flow compensation is gradient motion rephasing (GMR). This technique is very helpful in reducing flow-related artifact that may hinder evaluation of venous structures, particularly in the posterior fossa. These sequences also accentuate the visibility of vessels, especially where there is rapidly flowing blood. This is useful when the vein is narrow or where there is a thin rim of flow around the periphery of a thrombus.

\section{Sequence 5: Time-of-flight MRV of the sagittal sinus}

21. Run sequence 5 according to Table A2.3.6.

This sequence offers the possibility of demonstrating a venous angioma and showing its relationship to other major venous structures. 


\section{COMMENTARY}

\section{Background Information}

Venous angiomas are most often located adjacent to the frontal horns of the lateral ventricles or within the cerebellum. Most commonly small, their size ranges from microscopic to gigantic with involvement of an entire hemisphere. Unless large, they may be subtle on conventional angiography. These are usually more conspicuous on contrast-enhanced CT or MR images.

Venous angiomas are associated with focal neuronal abnormalities. The association with cavernous malformation is strong, and $7 \%$ of venous angiomas have associated cavernous malformations. Approximately one-third of cavernous angiomas are associated with venous angiomas.

Venous angiomas have no arterial components and are theorized to arise from an arrest of venous development after arterial development is complete (Dillon, 1997). This could cause retention of primitive medullary veins that are structurally intact but anomalous in location. Because they are physiologically normal, they are rarely associated with significant problems.

Venous angiomas are commonly found on contrast-enhanced cerebral examinations. While these patients commonly have headache, a causal relationship is unclear. Intracranial hemorrhage has been occasionally associated with venous angiomas.

Occasionally, the abnormal venous channel may be compromised by an adjacent structure, resulting in venous hypertension and parenchymal ischemia. Seizures are probably related to chronic ischemia. These changes can result in encephalomalacia and/or calcification. Patients with cerebellar cavernous angiomas have experienced ataxia, diplopia, and dizziness without demonstrable hemorrhage.

Grossly, there is a tuft of enlarged radially arranged anomalous medullary veins converging on a central draining vein which empties into a subependymal vein, cortical vein, or dural sinus. Microscopically, normal intervening brain parenchyma separates venous radicles, with venous walls being slightly thickened and hyalinized.

\section{Critical Parameters and Troubleshooting}

Use of intravenous contrast agent (gadolinium-DTPA) is essential when evaluating pa- tients for venous angiomas. Lesions of significant size may be overlooked if contrast-enhanced images are not obtained. Contrast-enhanced images should be obtained in patients who have cavernous angiomas to demonstrate associated venous angiomas. Post-contrast images should be performed in three planes (sagittal, coronal, and transverse).

\section{Imaging with MRV}

MRV may be helpful in evaluation of venous malformations. It is particularly important to identify a venous malformation when associated with a cavernous malformation. The venous malformation drains normal brain parenchyma and needs to be avoided by the neurosurgeon resecting the adjacent cavernous lesion (Rigamonti et al., 1998).

\section{Anticipated Results}

On pre-contrast images, flow voids and misregistration artifacts may be seen. Many venous angiomas may only be detected after injection of a contrast agent when the characteristic "Medusa head" appearance of venous radicles converging on a larger draining vein is seen (Truwit, 1992). When venous hypertension causes ischemia, $T_{2}$-weighted signal may be increased in adjacent parenchyma (Fischbein et al., 2000). The risk of hemorrhage rises with increasing venous pressure secondary to occlusion.

Transitional morphology may be present with lesions exhibiting arteriovenous shunting at the precapillary level.

The prognosis is generally excellent with no treatment required, except when associated with cavernous angioma or when there is some type of venous stenosis. In these cases, there is an increased rate of hemorrhage. Although a minority of lesions bleed, the rate of hemorrhage may be more frequent in the cerebellum (Rothfus et al., 1984).

\section{Time Considerations}

Although the actual scan time is $15 \mathrm{~min}$ and $46 \mathrm{sec}$, because of setup and injection time, we tell the patient that the examination will take approximately one-half hour.

\section{Acknowledgements}

The authors would like to thank the assistance from Shibu Mathew of Dallas VA Medical Center.
Cerebral Venous

Lesions

A2.3.11 


\section{Literature Cited}

Dillon, W.P. 1997. Cryptic vascular malformations: Controversies in terminology, diagnosis, pathophysiology, and treatment. Am. J. Neuroroentgenol. 18:1839-1846.

Fischbein, N.J., Dillon, W.P., and Barkovich, A.J 2000. Teaching Atlas of Brain Imaging, pp. 282 285. Thieme Medical Publishers, New York.

Glendhill, K., Moore, K.R., Jacobs, M., and Orrison, W.W., Jr. 2000. Chapter 21. In Neuroimaging (W.W. Orrison, Jr., ed.) pp.750-752. W.B. Saunders, Philadelphia.

Rigamonti, D., Spetzler, R.F., Medina, M., et al. 1998. Cerebral venous malformations. J. Neurosurg. 73:560-564.

Rothfus, W.E., Albright, A.L, Caey, K.F., et al. 1984. Cerebellar venous angioma: "Benign" entity? Am. J. Neuroroentgenol. 5:61-66.

Shellock, F.G. 2001. Pocket Guide to MR Procedures and Metallic Objects. Lippincott-Raven, Philadelphia.
Truwit, C.L. 1992. Venous angioma of the brain: History, significance, and imaging findings. Am. J. Roentgenol. 159:1299-1307.

\section{Key References}

Dillon, 1997. See above.

The author presents a broad overview of intracranial vascular malformations with regard to terminology, diagnosis, pathophysiology and treatment. Like opening Pandora's box we find things are more complicated than we had first thought.

Contributed by F. Allan Midyett

VA North Texas Health Care System

Dallas, Texas

Suresh Mukherji

University of Michigan

Ann Arbor, Michigan

Laurie Fisher

Siemens Uptime Service Center

Cary, North Carolina 